\title{
Anotaciones históricas sobre el Hospital de Enfermedades Infecciosas Francisco Javier Muñiz de Buenos Aires, Argentina
}

\author{
Enrique Laval
}

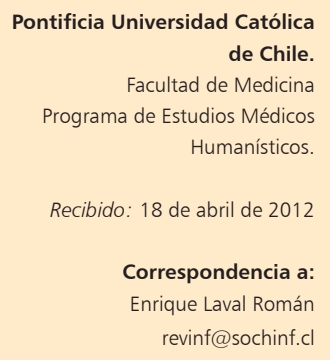

Correspondencia a: revinf@sochinf.cl

\author{
Historical notes on Infectious Diseases Hospital Francisco Javier Muñiz \\ in Buenos Aires, Argentina
}

The Infectious Diseases Hospital Francisco Javier Muñiz, Buenos Aires, Argentina, is the oldest in Latin America. It is over 100 years old and has a history worthy of pride. It became known as "Hospital of the pests" and was preceded by the old House of Insulation, which served as a quarantine station during epidemics of cholera, yellow fever and smallpox. The new House of Insulation, built in the neighborhood of Parque Patricios ("Barracks Hospital”), was renamed in 1904 in memory of Francisco Javier Muñiz, a former military doctor, naturalist and paleontologist. Its technical name is "Porteño Care Centre and National Reference Regional Infectious-Contagious Disease”. It receives numerous national and foreign undergraduate and postgraduate students in its Departments of Infectious Diseases and Respiratory Diseases.

Key words: Hospital, infectious diseases, epidemics, fellows training.

Palabras clave: Hospital, enfermedades infecciosas, epidemias, formación de especialistas.

\section{Introducción}

$\mathrm{E}$ l día 4 de septiembre de 2010, tuve la oportunidad de ser recibido en el Hospital de Enfermedades Infecciosas Francisco Javier Muñiz, por los doctores Rubén Masini y Augusto Fulgenzi, director y subdirector médico del establecimiento, respectivamente.

Durante toda una mañana conversamos sobre el hospital, visitando algunas dependencias, intercambiando impresiones relacionadas con la especialidad, evolución y cambios de la patología infecciosa, las últimas décadas, en nuestros países.

Al Hospital Francisco Javier Muñiz, concurren enfermos procedentes de toda América e incluso de los lugares más lejanos del planeta, lo que ha favorecido una visión amplia de la presentación de muchas enfermedades infecciosas.

En más de cien años de existencia, ha enfrentado todas las epidemias acontecidas en la Argentina. Por nombrar las más recientes, poliomielitis (1950), sarampión (1969) síndrome cardiopulmonar por hanta virus (1996), influenza porcina en 2009. Destaca, por supuesto, la pandemia de SIDA, ingresando el primer enfermo en 1984, cuando ya los médicos no esperaban enfrentarse con un flagelo de tales dimensiones. Como dijo Paul Cossedue, refiriéndose al Hospital, "su nombre es hoy casi inseparable del virus del SIDA”.

Las Cátedras de Enfermedades Infecciosas y de Tisioneumonología, que funcionan en el hospital, reciben diariamente a cientos de alumnos que tienen así la “opor- tunidad de asimilar los conocimientos y la experiencia de docentes de reconocida trayectoria”.

El nombre técnico del hospital lo define como "Centro Asistencial Porteño de Referencia Nacional y Regional de Enfermedades Infecto-contagiosas”. Es el más antiguo de toda Latinoamérica ${ }^{1-7}$.

\section{El aislamiento del enfermo contagioso}

"En el año 293 A.C. la peste asolaba Roma. Se enviaron mensajeros a Epidauro para pedir consejo. Esculapio ofreció su serpiente a los emisarios. Mientras remontaban el Tiber, aquella se deslizó del barco hasta una isla, indicando la voluntad de los dioses de que los romanos se trasladaran a ese lugar. Así lo hicieron y levantaron un templo, desapareciendo la epidemia”. Tal vez sea esta leyenda, una indicación de las opiniones y conjeturas, expresadas en aquel tiempo, de que los enfermos debían ser separados de los sanos ${ }^{8}$.

Una de las antiquísimas formas de evitar el contagio era la llamada “cuarentena”, significando que a los viajeros procedentes de una región infectada, se les mantenía aislados en un recinto alejado, para asegurarse de que si alguno estaba en el período de incubación de una enfermedad, tendría tiempo para desarrollarla. Si todos permanecían sanos, se les liberaba, ya que no constituían peligro para la población. Hoy se insiste que todos los viajeros procedentes de países con enfermedades de alta contagiosidad, posean certificado actualizado de vacunación correspondiente. 
Recordemos que el origen de la cuarentena se remonta a muchos siglos antes de la formulación de la teoría microbiana.

El 17 de enero de 1374, el vizconde Bernabé de Reggio, promulgó un decreto para evitar la introducción y diseminación de la peste, el cual imperfecto en su letra pero alentador en su espíritu, imponía un período de observación de 10 días, no surgiendo la relación etimológica con la palabra cuarentena. Este breve lapso sería extendido a 30, en 1377, por decreto del Concejo Municipal de Ragusa. Casi una década después, en Marsella y ante la sospecha de un brote pestífero, se establecieron los 40 días de aislamiento.

Muchos siglos antes de las comprobaciones experimentales, los hombres de ciencia, con sus conocimientos precarios, previeron la fundación de las "Casas de Aislamiento”, apareciendo los lazaretos y leprosarios ${ }^{1}$.

\section{La higiene en el Buenos Aires de 1871 y el aislamiento de los enfermos. El doctor José Penna}

Hacia 1871, Guillermo Rawson, había trazado un panorama desalentador, con respecto a la higiene que imperaba en la capital argentina, criticando el uso de basuras, desechos de las casas, materias vegetales y animales, para rellenar y nivelar las calles, “que fermentaban, escapaban por las capas porosas de la superficie y mezclándose con el aire, iba a ser respirado por los habitantes, constituyendo un fuente inagotable de veneno para la atmósfera”. La teoría de los miasmas no había muerto totalmente.

Gran parte de la población bebía agua de pozo y del río, que se vendía por las calles, extraída del Rio de la Plata, ciertamente contaminada por la población fluvial del puerto y por las materias animales líquidas procedentes de los mataderos establecidos en las riberas del llamado Riachuelo.

El sistema de letrinas era muy primitivo. Los receptáculos de materias excrementicias eran pozos comunes profundizados, casi siempre bajo el nivel de las aguas subterráneas. Otro factor importante de contaminación era la descarga en las aguas del Riachuelo de líquidos orgánicos, provenientes de los saladeros establecidos en sus orillas donde se faenaban 500.000 vacunos y dos millones de ovejas. Rawson infería que, como resultado de todas estas falencias, aguas estancadas y pantanos, se desencadenó en 1871, una de las más mortíferas epidemias de fiebre amarilla "que haya diezmado a un pueblo civilizado en todo el siglo XIX”. Ciento seis de cada 10.000 habitantes de Buenos Aires, murieron ese año y "cambiaron para siempre el perfil de ciertos barrios de la ciudad”.
Entonces las autoridades llevaron a cabo las obras para la provisión de agua potable y desagües, de las más adelantadas para la época.

Antecediendo a esta epidemia de fiebre amarilla, hubo otra importante en 1852-1853, además de una de cólera muy grande en $1868^{1,9}$.

\section{¿Cómo surgió en Argentina la primera Casa de Aislamiento?}

La ubicación austral de Buenos Aires no le permitía escapar de las pestilencias. La Quinta de Leinit, una casa situada en las calles Paraguay y Azcuénaga, construida por un médico de ese apellido, había servido de lazareto durante las epidemias de cólera de 1868 y la de fiebre amarilla, en 1871. Este lugar recibió su espaldarazo en 1882, durante una epidemia de viruela, por parte del Vicepresidente de la Comisión Municipal, el doctor José María Ramos Mejía, denominándola “Casa Municipal de Aislamiento".

La Casa de Aislamiento no tenía farmacia (utilizando aquello que le proveía el Hospital Buenos Aires), el personal era escaso, sus solas 40 camas eran desbordadas por las epidemias y en algunas ocasiones el número de enfermos ascendía a 95. El hacinamiento era impresionante, por lo que en mayo de 1883 se habilitaron dos carpas en el jardín, mientras que en agosto del mismo año, se alquiló un galpón contiguo que antes había sido una gasería.

El 17 de noviembre de 1883, el diario La Nación, publicó una nota dando cuenta de protagonistas y hechos trascendentales: "como lo anunciamos ayer existe en esta ciudad un enfermo de fiebre amarilla. Es un fogonero del vapor español Solís, llamado Vergara, el que fue internado en el Hospital Español. Informado el Intendente Municipal, hizo reconocer al enfermo por la Asistencia Pública, a cuyo director el doctor José María Ramos Mejía, le ordenó lo internara a ocho leguas de la ciudad y de la costa. El enfermo acompañado del doctor Penna, salió en una ambulancia en la cual iban la cama y los objetos usados por el paciente. En el paraje indicado el comisario Fernández había hecho armar dos carpas de 4 por 2,5 varas, en una de las cuales fue colocado el enfermo y en la otra el doctor Penna”. Este doctor Penna, al decir de Alois Bachmann, fue "uno de los apóstoles que, por su prédica y acción derramó infinitos beneficios para la salud de su pueblo" 10 .

Al finalizar 1884, desembarcaron en Buenos Aires, procedentes de Río de Janeiro, varios enfermos de fiebre amarilla. Los habitantes tenían todavía presente el estrago que aquella había causado trece años antes. Cundió el pánico. Pero otra vez surgió el esforzado trabajador heroico y Penna se aisló con los pestosos. Desde otro punto de vista, daría el puntapié inicial para una obra que lo perduraría: el hospital de infecciosos. José Penna avizoró el problema e instituyó la solución.

Un año antes el intendente Torcuato de Alvear había 
adquirido un terreno delimitado por las calles Entre Ríos, Matheu, Camino Alsina y Patagones, donde comenzaron a construirse dos grandes galpones de madera con techos de tejas, ubicados paralelamente, separados entre sí por 15 metros y luego otro cuadrado de 16 metros de lado. El predio era, aunque no para la época, de grandes dimensiones: 5 manzanas. Los dos pabellones contiguos fueron divididos y con la construcción de uno más, aprovechando una armazón de fierro situada en Alsina y Perú, se conformaron cinco salas. Terminaba 1888 cuando se le agregó una estación sanitaria con una estufa de desinfección. Ese mismo año, otra epidemia de cólera obligó a construir 4 ó 5 salas más, siempre de madera. La nueva Casa de Aislamiento recibió otra prueba de fuego, saliendo airosa, apareciendo en las memorias municipales con el nombre de Hospital de Barracas. Su construcción definitiva, comenzaría oficialmente en agosto de 1894.

Fracasadas las tentativas del intendente Antonio Crespo para fundar otro hospital para aislamiento de pacientes infecciosos, adquiriendo el terreno donde actualmente se levanta el Hospital Tornú (Combatientes de Malvinas 3002. Villa Urquiza. Buenos Aires), el director de la Asistencia Pública, José Ayerza en 1883, nombró una comisión que debería proyectar el nuevo hospital. Ya no se pensaba en una nueva fundación ni en su traslado, sino atinadamente mejorar el lugar donde la Casa de Aislamiento se encontraba situada.

La idea primigenia fue levantar tres hospitales aislados entre sí, con comunicación por medio de galerías incompletas, con servicios accesorios individuales. Esto dio lugar a una sección de 18 pabellones, otra de 5 y la última de 14. El proyecto había cobrado inusitada importancia. Encontrándose la obra en plena ejecución, se adquirieron dos terrenos que tenían su frente sobre la Avenida Armancio Alcorta, que darían lugar a los pabellones Roberano y Santa Ana (luego salas 22 y 23), destinados a enfermos tuberculosos.

El 30 de junio de 1900 se fundó la Cátedra de Clínica Epidemiológica, cuyo primer profesor fue José Penna y el 20 de septiembre de 1901, la Escuela Interna para la educación de los niños hospitalizados por enfermedades infecciosas.

El 20 de octubre de 1904, la ex-Casa de Aislamiento, paso a denominarse Hospital de Enfermedades Infecciosas Doctor Francisco Javier Muñiz.

A la muerte de José Penna, en 1919, fue designado Profesor Titular de Clínica Epidemiológica y Director Técnico de todos los servicios del hospital el doctor Francisco Destéfano. Bajo el impulso del Director, doctor Carlos N. Pico, en 1930, el hospital es remozado totalmente, creándose nuevas dependencias. Se habilita en 1932 el Pabellón Koch y el 8 de octubre de 1937, se crea la Cátedra de Clínica y Patología de la tuberculo$\operatorname{sis}^{1,3,4,10,11}$.

\section{El doctor Francisco Javier Muñiz}

Francisco Xavier Thomas de la Concepción Muñiz, nació en Montegrande, provincia de Buenos Aires, el 21 de diciembre de 1795. A los 11 años, en 1807, se alistó como cadete en el Regimiento de los Andaluces, siendo herido mientras intervenía en la defensa de Buenos Aires, durante las invasiones inglesas.

Se tituló de medico en 1822, después de cursar sus estudios en el Instituto Médico Militar, cuando ya era parte de la Universidad de Buenos Aires. Recién en 1844, Muñiz recibió el doctorado.

Además de ser médico notable, su figura destacó claramente en las ciencias naturales, especialmente en la paleontología, recogiendo y reconstruyendo fósiles.

Bernardino Rivadavia lo nombró médico y cirujano principal, a cargo del servicio de ambulancias y hospital, con el grado de teniente coronel, en 1826, al estallar la guerra contra Brasil.

Durante una epidemia de escarlatina en Buenos Aires, 1836-1837, su experiencia clínica quedó reflejada, en la "Descripción y curación de la fiebre escarlatina”, publicada en 1844, en la Gazeta Mercantil.

Después de haber atendido más de 300 enfermos de carbunco, admitió la existencia de un agente infeccioso contagiante, distinguiendo dos formas clínicas: la pústula maligna localizada y la septicemia carbuncosa.

En 1841, Muñiz le "regaló" su colección paleonto-

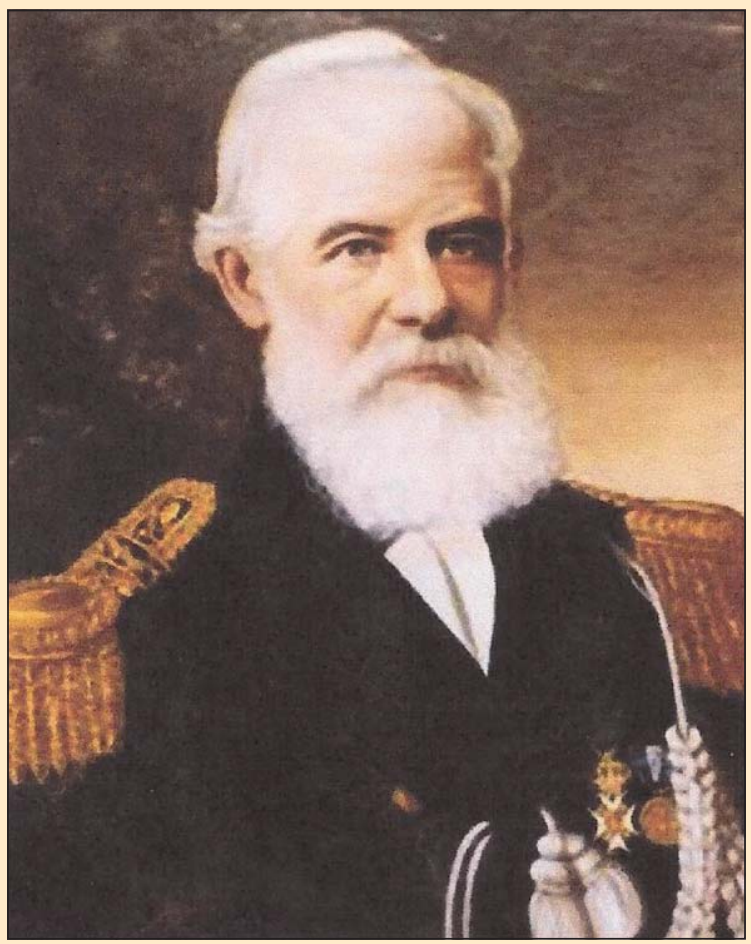

Figura 1. Doctor Francisco Javier Muñiz (1795-1871). 
lógica al Dictador Rosas, quien a su vez la obsequió al almirante francés Dupotal. Para algunos, se trató de un despojo ya que habría sido una donación “obligatoria”. Después de reunir una nueva colección, la donó al Museo de Buenos Aires, en 1857.

Buenos Aires quedó desprovisto de vacuna antivariólica en 1844. En un gesto de altruismo, Muñiz se trasladó a la capital con una de sus hijas, de pocos meses y recién vacunada, con cuya linfa pudieron ser inoculadas más de 20 personas, noble actitud que permitió restablecer la vacunación.

En 1850 fue nombrado catedrático de Partos, Enfermedades de Mujeres y de Niños. El voto de la mayoría de sus pares lo llevó a la Presidencia (Decano) de la Facultad de Medicina. Fue Diputado Provincial por Luján en 1853 y al año siguiente Senador.

Ya retirado, pero impulsado por su sentido del deber regresó a Buenos Aires a trabajar, donde contrajo fiebre amarilla durante la epidemia que hemos mencionado, falleciendo el 8 de abril de 1871.

$\mathrm{Al}$ decir de Mitre "murió como mártir al pie de la bandera de la caridad"12-15.

\section{Médicos chilenos becados en el Hospital Muñiz}

Profesor Doctor Roque Kraljevic Orlandini. Gracias a una beca de estudio y perfeccionamiento en enfermedades infecciosas y salud pública, de la Dirección de la Honorable Junta de Beneficencia y Asistencia Social, se incorporó en abril de 1942 y hasta octubre de ese año, a la Cátedra de Enfermedades Infecciosas del Profesor Titular doctor Carlos Fonso Gandolfo, en el Hospital Muñiz.

Era el campo ideal para que un clínico joven aprendiera de primera mano y al lado del enfermo, la enorme variedad de procesos infecciosos. Participó activamente como alumno del Curso para Especialistas en Enfermedades Infecciosas (primer médico chileno graduado en la especialidad). Los conocimientos adquiridos le sirvieron para delinear a futuro en Chile, los programas docentes. Su estadía en el Hospital Muñiz, le permitió conocer el más importante de la especialidad en América Latina, estudiando su organización, así como la de las cátedras de enfermedades infecciosas, funcionamiento, criterios y fuentes bibliográficas.

Doctor Jaime Vitali Cáceres. Completó sus estudios médicos en la Facultad de Medicina de Buenos Aires, el año 1974. Posteriormente, durante 1975 y 1976, efectuó estudios de postgrado, en el Hospital Muñiz, bajo la dirección del Profesor Omar J. Palmieri, obteniendo el título de Especialista en Enfermedades Infecciosas.

Llegó a Chile en 1978, revalidando su título de médico cirujano, ingresando en 1980 al Hospital de Enfermedades
Infecciosas Prof. Dr. Lucio Córdova de Santiago, formando parte de su equipo docente-asistencial. Actualmente es Jefe de la Unidad de Pacientes Críticos.

Recuerda con cariño y admiración la estadía en el Hospital Muñiz, donde se impregnó de la disciplina y experiencia en el manejo de la patología infecciosa ${ }^{16,17}$.

\section{"El Muñiz hoy"}

Deseo terminar estas “anotaciones históricas” sobre el Hospital Francisco Javier Muñiz, destacando el nombre de su revista de divulgación científica, cuyo volumen $n^{\circ}$ 1 corresponde al año 1998 y el nº 14, al recién pasado 2011. Cumple con la misión de publicar la rica experiencia del quehacer clínico y asistencial, tanto de las Cátedras de Enfermedades Infecciosas, lideradas por connotados especialistas titulares y adjuntos, así como las de los médicos dedicados a neumotisiología.

"En la actualidad es un hospital monovalente dedicado a las enfermedades infecciosas, con casi 300 camas disponibles (años atrás tuvo más de 1.000), con prevalencia de pacientes afectados de tuberculosis y/o SIDA. Durante el año 2010 fueron asistidos 4.445 pacientes en internación. No cuenta con un área programática delimitada, ya que no es un Hospital General de Agudos, por lo que su zona de influencia se extiende más allá de las fronteras del barrio Parque Patricios, constituyéndose como centro de referencia de nivel nacional e internacional”. Con estas palabras inicia el Ministerio de Salud bonaerense, la caracterización de lo que sería hoy el antiguo y más que centenario hospital.

Es el centro con el mayor número de pacientes seropositivos para VIH ambulatorios, más de tres mil enfermos bajo tratamiento anti-retroviral continuo, seguidos mensualmente. Existen múltiples instancias de investigación para estos enfermos, así como cualesquiera otros con

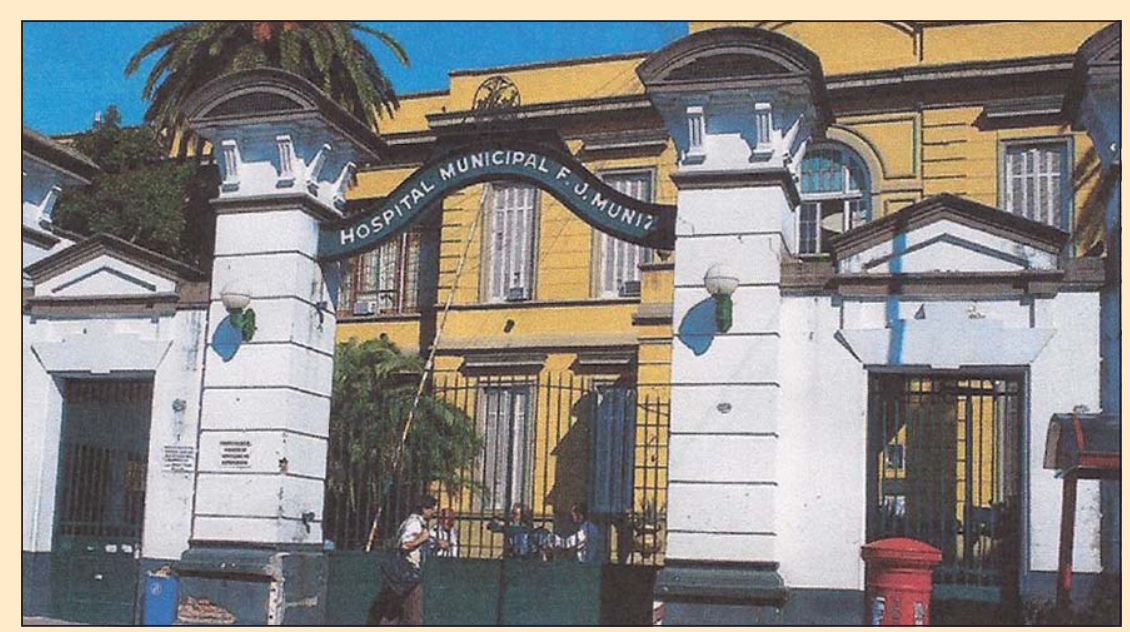

Figura 2. Hospital Francisco Javier Muñiz. Frontis actual. 
procesos infecciosos de diversas etiologías, que deban estudiarse y tratarse.

Este hospital cuenta con el Departamento de Atención Intensiva al Paciente Infeccioso Crítico, compuesto por cuatro salas de terapia intensiva de distinta complejidad. Una de ellas es la única en el sistema, que posee presión negativa para la atención de pacientes inmunocomprometidos y otra orientada a patologías respiratorias, todas con perfil infectológico.

En lo que se refiere a la asistencia en la internación de enfermos afectados por VIH/SIDA, el hospital tiene cuatro salas de adultos (una de ellas de mujeres) con 65 camas y una para niños con 15 camas. Dentro del actual Departamento de Infecciosos, se encuentran dos salas de internación general de enfermedades infecciosas, una sala de hospitalización de hepatopatías, una de patologías regionales y tropicales. Otra de pediatría para enfermedades infecciosas.

Junto con la intensa actividad docente se incluyen rotaciones y pasantías, tanto para alumnos residente en el país, como en el extranjero.

No quisiera concluir sin referirme a la creación del Museo del Hospital, en octubre de 2008, por decisión de las autoridades directivas, con el fin de proteger, preservar y promover el patrimonio arquitectónico e históricocultural del establecimiento.

En el evento "Las noches de los museos" de Buenos Aires, el 14 de noviembre de 2009, el Museo del Hospital expuso parte de su notable colección de fotografías que plasmaron los primeros pabellones y quehaceres cotidianos de la institución.

Los medios de prensa descubrieron una faceta poco conocida del primer Hospital de Enfermedades Infecciosas de la República Argentina, “convertido a fuerza de logros científicos y asistenciales, en un hito de la historia de la Salud Pública argentina”18,19.

Agradecimientos. A la Sra. Magdalena Cox, secretaria de la Embajada de Chile en Buenos Aires, por su colaboración en la búsqueda del material bibliográfico.

\section{Resumen}

El Hospital de Enfermedades Infecciosas Francisco Javier Muñiz, de Buenos Aires, República Argentina, es el más antiguo de América Latina, con más de 100 años de existencia y una historia digna de orgullo; fue conocido como "Hospital de las pestes". Antecedido por la antigua Casa de Aislamiento, que sirvió de lazareto durante epidemias de cólera, fiebre amarilla y viruela. La nueva Casa de Aislamiento, construida en el barrio Parque Patricios (“Hospital de Barracas”), pasó a denominarse en 1904,
Hospital Francisco Javier Muñiz, por quien fuera médico militar, naturalista y paleontólogo. Su nombre técnico es "Centro Asistencial Porteño de Referencia Nacional y Regional de Enfermedades Infecto-Contagiosas" y recibe a numerosos alumnos nacionales y extranjeros, en sus Cátedras de Enfermedades Infecciosas y de Tisioneumología, para docencia de pre y post-grado.

\section{Referencias bibliográficas}

1.- Pérgola F. El Hospital Francisco Javier Muñiz. Antecedentes para su historia. Rev Arg Infect 1994, 7: 4-13.

2.- Martino O. Perspectiva histórica del Hospital Francisco Javier Muñiz. El Muñiz Hoy 2004; 7: 94-9.

3.- Botánico Sur. http://www.botanicosur.com.ar/periodicos/ per_23htal1\%20muñizhtml. (consultado el 21/9/2010).

4.- Cosseddue P. Hospital de Infecciosas Dr. Francisco Muñiz. http://www.botanicosur.com.ar/periodicos/per_23htal1\%20 muñizhtml. (consultado el 21/9/2010).

5.- Hospital Muñiz. http://es.wikipedia.org/wiki/Hospital_ Mu\%C3Bliz (consultado el 21/9/2010).

6.- Comité de Redacción. Editorial. El Muñiz Hoy 2004; 7: 1.

7.- Palmieri O J. Enfermedades Infecciosas. Impta. Grefinor

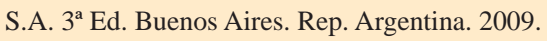

8.- $\quad$ Laval R E. De la cuarentena. Rev Chilena Infectol 1984; 1: 54-5.

9.- Cartwright F, Biddiss M. Grandes pestes de la historia. Ed. El Ateneo. Buenos Aires. Rep. Argentina. 2005.

10.- Capurro E, Cheri A. José Penna. Rev. Arg. Leprol. 1966, 3:57-58.

11.- Gueverino A. Francisco Destéfano. 1879-1950. Semblanza de un realizador. Mundo hospitalario 1969; 215: 19-20.

12.- Aidar O, Arechavala A, Ralli H, Vescovo M, Zabala O H, Fulgenzi A. Francisco Xavier Thomas de la Concepción Muñiz. Héroe de la Independencia, Médico y Naturalista. El Muñiz Hoy 2000; 3: 119-23.

13.- Francisco Javier Muñiz. http://es.wikipedia.org/wiki/ Francisco_Javier_Mu\%C3Bliz. (consultado el 21/9/2010).

14.- Palmero D. ¿Quién fue el Prof. Dr. Francisco Javier Muñiz? Ministerio de Salud. Buenos Aires. Rep. Argentina. 2010.

15.- Francisco Javier Muñiz. Un médico con espíritu paleontólogo. http://www.grupopaleo.com.ar/paleoargentina/ pionero.0.4htm (consultado el 21/9/2010).

16.- Kraljevic O R. Recuerdos de un viejo infectólogo. Contribución a la Historia de la Infectología en Chile (19371970). Santiago de Chile. 1998. Sin pie de Impta.

17.- Vitali C J. Comunicación personal.

18.- Ministerio de Salud. Buenos Aires. Rep. Argentina. Hospital de Infecciosas Francisco Javier Muñiz. 2010.

19.- Pizarro N J. El Museo del Hospital de Enfermedades Infecciosas Dr. Francisco Javier Muñiz, en "La Noche de los Museos”. El Muñiz Hoy 2010; 13: 17-8. 